\title{
Chi comanda? La fonte della moralità in Kant e Levinas
}

\author{
Edvaldo Antonio de Melo* \\ Cristiane Pieterzack ${ }^{* *}$
}

DOI 10.20399/P1982-999X.2016v1n2pp28-46

\begin{abstract}
Resumo
Neste artigo, buscaremos colocar em relação Levinas e Kant, pondo-se a seguinte questão: qual é a fonte da moralidade? Em outras palavras: quem é responsável? Para fins de clareza metodológica o texto será dividido em três partes nas quais internamente tomaremos para reflexão o exame da Fundação a metafísica dos Costumes (Grundlegung zur Metaphysik der Sitten) de Kant e Humanismo do outro Homem (Humanisme de l'autre homme) de Levinas. Estas obras nos permitirão apresentar as ideias para um possível ligame entre os dois pensadores. Procuraremos mais exatamente refletir, por uma parte, como Kant funda a moralidade; e por outra, como realiza-se a passagem da intencionalidade ao ético em Levinas.
\end{abstract}

Palavras-chave: Autonomia; Heteronomia; Proximidade; Responsabilidade.

\begin{abstract}
In this text we will try to relate Levinas and Kant by facing the question: what is the source of morality? In other words: who's in charge? For methodological clarity, the text will be divided into three parts in which we would take into consideration Fondazione della metafisica dei costumi (Grundlegung zur Metaphysik der Sitten) by Kant, and Umanesimo dell'altro uomo (Humanisme de l'autre homme) by Levinas that will allow us to present some ideas for a possible link between the two thinkers. We will seek more accurately to reflect, on the one hand, as Kant founds morality; on the other hand, as it gives the passage from intentional to ethical in Levinas.
\end{abstract}

Key-words: Autonomy; Heteronomy; Proximity; Responsability

\section{Introduzione}

Il testo è diviso in tre momenti. Nel primo momento si approfondisce la questione sul fondamento della morale a partire dalla seconda sezione della Fondazione della metafisica dei costumi di Kant, intitolata: "Passaggio dalla filosofia morale popolare alla metafisica dei costumi". Fare questo "passaggio" significa andare dalla regola pura soggettiva dell'agire alla pura forma di una legge universale. Questo itinerario kantiano ha come scopo presentare con chiarezza la facoltà pratica razionale che lui stesso chiama "ragione pratica".

Nel secondo momento, verrà analizzata in modo più specifico, l'opera Umanesimo dell'altro uomo ${ }^{1}$ di Levinas pubblicata quasi due secoli dopo la Fondazione della metafisica dei costumi. Levinas elabora una profonda critica ai precedenti umanesimi. La critica levinassiana deriva dal fatto che gli umanesimi precedenti risultano insufficienti e incompleti nel loro tentativo di definire e proteggere l'essere umano, tanto è vero che non avranno abbastanza forza per impedire alcuna offesa o aggressione contro l'uomo singolo e intere popolazioni, per non parlare della più eclatante delle barbarie che la storia ha conosciuto: il nazismo.

\footnotetext{
* Dottorando in Filosofia alla Pontificia Università Gregoriana (Roma). Borsista CAPES - Brasile. Per contato, e-mail: edvaldoantonio87@gmail.com

** Dottoranda in Filosofia alla Pontificia Università Gregoriana (Roma). Per contato, e-mail: ircris@ hotmail.com

${ }^{1}$ Umanesimo dell'altro uomo è stato pubblicato in 1972, ma ha preso forma dalle varie conferenze che Levinas ha pronunciato dal 1961 a 1963.
} 
Dopo aver esposto brevemente il pensiero di Kant e Levinas, si cercherà di metterli a confronto. L'interesse ad esaminare la loro relazione è aumentato notevolmente. Infatti, negli ultimi anni, sono stati scritti diversi saggi interessanti che collegano il pensiero di Levinas all'etica kantiana.

\section{Dalla filosofia morale popolare alla metafisica dei costumi}

Nella Critica della ragione pura (1781) Kant aveva studiato la possibilità e la legittimità di una legislazione a priori dello spirito. Nella Fondazione (1785), invece, lui parte dai giudizi della coscienza comune e cerca il principio razionale che li fonda. Nella Fondazione ci sono praticamente tutti gli elementi essenziali della morale kantiana, però solo successivamente, nella Critica della ragione pratica (1788), Kant mette in evidenza la moralità a partire dalla funzione pratica della ragione.

In questa prima si farà un'esposizione generale riguardante la premessa essenziale del concetto di dovere come adeguamento della massima alla pura forma di una legge universale. In un secondo momento, verrà dimostrato come avviene il passaggio dalla filosofia morale popolare alla metafisica dei costumi, mettendo in evidenza gli imperativi. A titolo d'introduzione, possiamo dire che la domanda principale può essere espressa così: Come è possibile l'imperativo categorico? Davanti a questa domanda cercheremo di capire qual è il fondamento della necessità assoluta (categorica) dell'imperativo morale.

\subsection{La determinazione del concetto di dovere}

Premessa essenziale della "metafisica dei costumi" è la determinazione del concetto di dovere come adeguamento della "massima" alla pura forma di una legge universale $^{2}$. Come capire la necessità che la massima sia conforme al dovere? Per fare questo adeguamento, la ragione si trova davanti ad alcune difficoltà in relazione alle inclinazioni della sensibilità umana. E allora si fa ricorso alla "costrizione" della volontà non buona alla ragione che Kant chiama "imperativo", regola o termini che esprimono un "dover essere" (Sollen).

Per capire questo itinerario attraverso il quale la volontà viene costretta alla ragione, bisogna ricordare ciò che è stato detto nella prima sezione della Fondazione, ossia, che si tratta del "passaggio dalla comune conoscenza morale di ragione alla conoscenza filosofica" 3 . Si cercherà di precisare alcuni concetti presenti nella prima sezione e che sono molto importante per la seconda, come quelli di "volontà buona" e di "dovere".

Per Kant, la volontà buona è la pietra preziosa che brilla da sé ${ }^{4}$ La volontà guida tutto, in modo che il vero compito della ragione è quello di produrre una volontà che non sia mezzo per il compimento di altro scopo, ma buona in sé stessa. Dunque, per sviluppare il concetto di volontà buona, Kant esamina il concetto di dovere, cioè, "la necessità di un'azione per rispetto alla legge" 5 .

Questa definizione di dovere viene da due principi kantiani: 1) l'azione conforme al dovere al quale il soggetto possiede una "immediata" inclinazione ${ }^{6}$ e 2 )

\footnotetext{
${ }^{2}$ Cf. GONNELLI, F. "Introduzione". In: KANT, I. Fondazione della metafisica dei costumi. Bari: Laterza, 2009, p. xiv.

${ }^{3}$ KANT, 2009, p. 15-41.

${ }^{4}$ Cf. Ibidem, p. 17. Per Kant, come se verrà più avanti, la volontà è la ragione pratica (Ibidem, p. 57).

${ }^{5}$ Ibidem, p. 29.

${ }^{6}$ Cf. Ibidem, p. 23.
} 
l'azione compiuta per dovere, dove il valore morale non si trova nel mezzo, ma nella massima in base alla quale viene decisa ${ }^{7}$.

Dopo aver chiarito il concetto di dovere e di buona volontà diviene più facile concepire la premessa metodologica della Metafisica dei Costumi nella quale il concetto di dovere diventa un adeguamento alla massima. Questo presuppone capire la Metafisica dei costumi come la dottrina che ha il suo fondamento in un principio supremo di moralità a priori, distinto da ogni impulso empirico o sensibile e separata dall'antropologia. Adesso possiamo approfondire la sezione seconda nella quale Kant mette in evidenza il "passaggio" dalla filosofia morale popolare alla metafisica dei costumi. Come affronta Kant la questione del dovere in questa seconda sezione?

All'inizio della suddetta sezione, Kant continua a sviluppare il concetto di dovere. Secondo lui, è vero che tale concetto deriva dall'uso comune della ragione pratica, però non è un concetto empirico dell'esperienza perché non può conferire universalità e necessità. Anche nella Critica della ragione pura, quando parla delle idee in generale, critica coloro che accusano la Repubblica di Platone d'essere impraticabile ${ }^{8}$.

Contrario a questi pensieri, ciò che per Kant caratterizza l'ideale della ragione è il "dover essere" e non l'esperienza. L'azione morale viene condannata per il dovere e non per l'interesse delle inclinazioni umane. Lui stesso dice: "In realtà, attraverso l'esperienza è assolutamente impossibile stabilire con piena certezza anche un solo caso in cui la massima di un'azione per ogni verso conforme al dovere abbia riposato esclusivamente su fondamenti morali e sulla rappresentazione del proprio dovere" .

Kant continua dicendo che "quando si tratta del valore morale non sono in questione le azioni, che si vedono, ma quei loro principi interni, che non si vedono"10. Anche se nessuna esperienza può provare l'esistenza di un atto puramente morale $\mathrm{e}$ anche se si può dubitare dell'esistenza di tale atto, ci resta concludere che il dovere è un'esigenza della "ragione pura". Chi comanda l'azione morale è il dovere e non l'interesse delle inclinazioni, ossia, è la ragione che determina la volontà per mezzo di principi a priori.

Accanto al concetto di dovere e di volontà bisogna aggiungere che il significato di "legge" acquisisce grande importanza nella morale kantiana. Se da una parte esiste la "massima" come principio soggettivo dell'agire, dall'altra esiste la "legge" pratica come principio oggettivo valido per ogni essere razionale e fondamento dell' agire, ossia un imperativo $^{11}$.

Per Kant, ogni esempio deve essere giudicato secondo i principi della moralità e non dell'esperienza, "non si potrebbe neppure immaginare qualcosa di peggio per la moralità, che volerla trarre da esempi" 12 . In questo senso, persino il "santo" del Vangelo deve essere in primo luogo paragonato con l'ideale della perfezione morale, prima che lo si riconosca come tale ${ }^{13}$. Si può anche dire che Dio come sommo bene viene dall'idea

\footnotetext{
${ }^{7}$ Cf. KANT, 2009, p. 29.

${ }^{8} \mathrm{Cf}$. KANT, I. Critica della ragion pura. Milano: Bompiani, 2007, B 372-373.

${ }^{9}$ KANT, 2009, p. 43.

${ }^{10}$ Ibidem, p. 45.

${ }^{11}$ Cf. Ibidem, p. 75 (nota).

12 Ibidem, p. 47.

13 Nel "santo" a modo kantiano, c'è una conformazione della volontà alla ragione senza la costrizione della stessa ragione; perciò la domanda "Maestro buono, che cosa devo fare per avere in eredità la vita eterna?" (secondo Mc 10,17; Lc 18,18) rimane stimolante. Sembra che per Kant l'amore viene inteso nel senso della moralità e così sorge una domanda che tocca alla filosofia della religione: in Kant la religione sarebbe ridotta alla moralità?
} 
della perfezione morale che la ragione traccia a priori e che connette inscindibilmente con il concetto di una volontà libera ${ }^{14}$.

E così, non si può fondare la morale sull'esperienza perché, secondo la critica di Kant, la sola "filosofia morale popolare" 15 , senza alcun autentico principio supremo $a$ priori della moralità indipendente da ogni esperienza, non sarebbe possibile distinguere la conoscenza "comune" da quella "filosofica". Perciò, che cos'è necessario per "fondare" la metafisica dei costumi? Per Kant la prima cosa da fare è fondare la dottrina dei costumi sulla metafisica, e successivamente, quando è ben salda su di essa, cercare di darle accessibilità attraverso una esposizione popolare ${ }^{16}$. In questo senso la Metafisica dei costumi può essere considerata una dottrina fondata sui principi interamente a priori, cioè, esclusivamente sui puri concetti di ragione libera da ogni empiria non mescolata a nessuna antropologia, né teologia, ancor meno fisica o iperfisica $^{17}$.

Riguardo alla premessa metodologica essenziale, risultano evidenti alcune "conclusioni" 18: i concetti morali hanno la loro sede e la loro origine interamente $a$ priori nella ragione e non possono essere dedotti per astrazione da alcuna conoscenza empirica puramente contingente. Nella purezza dell'origine dei concetti sta appunto la loro dignità, così da servire per noi da supremi principi pratici come valore illimitato della ragione.

Siamo così arrivati a un punto fondamentale e che corrisponde al cuore della seconda sezione, cioè, al punto in cui Kant presenta con chiarezza la facoltà pratica razionale, ossia, la volontà. E allora, si può fare il "passaggio" dalle regole di determinazione generale fino al concetto del dovere.

\subsection{Il comando dell'imperativo morale}

Per chiarire il comando dell'imperativo morale kantiano, mettiamo in evidenza ciò che lui stesso presenta come fossi la facoltà pratica razionale nell'uomo: "Ogni cosa della natura opera secondo leggi. Solo un essere razionale ha la facoltà di agire secondo la rappresentazione delle leggi, vale a dire secondo i principi, ovvero ha una volontà. Poiché per la derivazione delle azioni dalle leggi è richiesta la ragione, allora la volontà non è altro che la ragione pratica" ${ }^{19}$. La morale kantiana riguarda la legge universale, ove la massima è conforme al dovere. Ora, questo va bene quando la volontà sceglie "solo ciò" che la ragione riconosce come praticamente necessario, come buono.

Che cosa succede però quando la ragione da sola non determina la volontà? La volontà non è perfetta a cause delle condizioni soggettive, allora può darsi che ci possa essere conflitto tra la ragione e le condizioni soggettive nel momento della determinazione della volontà. E perciò avviene una "costrizione" della volontà. Questa costrizione non è naturale e così le leggi della ragione sono presentate alla volontà come comandamenti, "imperativi". Si può anche dire: se la ragione per sé sola non determina la volontà, allora le azioni che oggettivamente vengono riconosciute come necessarie, sono soggettivamente contingenti.

\footnotetext{
${ }^{14}$ Cf. KANT, 2009, p. 49

15 Secondo Kant, il termine Popularität, va inteso nel senso della Popularphilosophie, la filosofia scritta in linguaggio non specialistico, accessibile al grande pubblico, praticata da alcuni illuministi berlinesi (Cf. "Le note al testo italiano". (Ibidem, p. 168, nota 5).

${ }^{16}$ Cf. Ibidem, p. 49.

${ }^{17}$ Cf. Ibidem, p. 51.

${ }^{18}$ Cf. Ibidem, p. 53 e 55.

${ }^{19}$ Ibidem, p. 55 e 57.
} 
Secondo Kant, questo non succede con la volontà divina perché è una volontà "santa": obbedisce alla legge razionale senza una costrizione. Perciò, per la volontà divina non sono necessari imperativi. Il "volere" di Dio è già per se stesso necessariamente concorde con la legge. Una volontà perfettamente buona che si trova sotto leggi oggettive (del bene) può determinarsi da sé attraverso la rappresentazione del bene. In questo senso, per la volontà divina (una volontà santa) non valgono imperativi, perché il "volere" è d'accordo con la legge.

Che cos'è un imperativo? Come se distingue? Quali sono le sue possibilità? Come si arriva alla formula dell'imperativo universale del dovere? Possiamo dire che queste domande costituiscono lo scopo di questo nostro lavoro. Per Kant gli imperativi sono formule di determinazione dell'azione che vengono espressi attraverso un "dover essere" e indicano così il rapporto di una legge oggettiva della ragione con una volontà.

Gli imperativi possono comandare in modo ipotetico o categorico. Gli imperativi ipotetici rappresentano la necessità pratica di un'azione possibile come mezzo per ottenere qualcos'altro che si vuole e riguardano la materia dell'azione e ciò che da questa si debba conseguire. Negli imperativi ipotetici c'è uno scopo "possibile" o "reale".

Possiamo dire che uno scopo è "possibile" quando il principio è "problematicamente" pratico. Facciamo uso di alcuni esempi citati da Kant: i precetti del medico al paziente per guarire e la preoccupazione dei genitori di insegnare tutto ai loro bambini. Questi esempi sono anche chiamati "imperativi dell'abilità" perché si riferiscono all'uso dei mezzi per ottenere ogni genere di fine "desiderabile".

Si dice che uno scopo è "reale" quando il principio è "assertoriamente" pratico. Kant parla, per esempio, dello scopo della felicità. Che cos'è la felicità? Secondo lui, la felicità è una necessità naturale che si può "presupporre" con certezza a priori in ogni uomo. In questo imperativo, c'è l'abilità della "prudenza" 20 .

L'imperativo categorico rappresenta un'azione senza riferimento ad un altro fine, come in sé stessa oggettivamente necessaria che riguarda la "forma", il principio dell'azione stessa e il Bene per essenza. Secondo Kant, l'imperativo categorico può essere chiamato di "principio della moralità". Vale come un principio "apoditticamente" pratico.

Rispetto alla relazione volontà e principi, Kant parla di diseguaglianza nella costrizione della volontà. Secondo lui essi sono: o "regole" dell'abilità, denominati imperativi "tecnici" (propri dell'arte); o sono "consigli" della prudenza, nei casi degli imperativi "pragmatici" (propri del benessere); o "comandi morali" (propri del libero agire in generale, ossia dei costumi).

Di fronte alla questione della possibilità degli imperativi, vale precisare che per Kant solo la legge implica il concetto di una necessità incondizionata, oggettiva e universalmente valida. Il "consiglio", per esempio, contiene, certo, necessità, ma può valere soltanto sotto la condizione soggettivamente contingente. L'imperativo categorico, al contrario, non è limitato da alcuna condizione e in quanto assolutamente pratico, necessario, può essere chiamato comando. La domanda sulla possibilità di tutti gli imperativi, secondo Kant fa riferimento al "come" possa essere pensata la semplice costrizione della volontà che l'imperativo esprime nel compito.

Per quanto riguarda l'imperativo "dell'abilità", secondo Kant, chi vuole il fine vuole anche il mezzo a ciò inderogabilmente necessario, che è in suo potere. La proposizione riferente a questo imperativo è chiamata "analitica", ossia, il fine è solo

${ }^{20}$ Secondo Kant la parola "prudenza" viene intesa in un duplice senso: come prudenza mondana, cioè l'abilità di un uomo nell'avere influsso sugli altri (per i suoi scopi); e nel senso di prudenza privata, cioè sapere comprendere come unificare tutti questi scopi a proprio vantaggio (cf. KANT, 2009, p. 63, nota). 
possibile perché nel volere un oggetto come effetto da me prodotto viene pensata già la mia causalità come causa agente nell'uso dei mezzi. Per quanto riguarda gli imperativi della "prudenza", rispetto al concetto della felicità, questo è indeterminato. Sebbene ogni uomo desideri raggiungerla, nessuno tuttavia determina mai cosa davvero desidera e vuole. Gli elementi che appartengo al concetto della felicità sono empirici e, tuttavia, l'idea di felicità esige un tutto assoluto ${ }^{21}$.

Come è possibile l'imperativo categorico? Siamo davanti alla domanda principale della Fondazione, perché si tratta di comprendere la necessità assoluta del comando dell'imperativo morale. Secondo Kant è senza dubbio l'unica questione che esige una risposta. Però, come dice lo stesso Kant, "ma qui non bisogna in nessun modo perdere di vista che non si può stabilire per mezzo di alcun esempio, quindi empiricamente, se in generale, si dia un tale imperativo"22.

Questa "condizione senza esempi" di Kant per la possibilità dell'imperativo categorico, ossia, di non poter appoggiarsi, come negli imperativi ipotetici, ad alcun presupposto, comporta una difficoltà perché va inteso come una proposizione sinteticopratica $a$ priori $^{23}$. Che cos'è questo "sintetico"?

Il "sintetico" qui viene inteso come non derivato di "qualcos'altro" come si può vedere negli ipotetici. È vero che l'espressione "proposizione sintetico-pratica a priori" comporta una difficoltà nella conoscenza teoretica, ma si può dedurre che non ne comporterà meno in quella pratica. La sinteticità dell'imperativo categorico riguarda lo specifico della condizione di possibilità ${ }^{24}$. Allora, come capire che tale imperativo contiene solo la necessità che la massima sia conforme alla legge universale?

Come accennato prima, per capire lo spirito dell'imperativo categorico bisogna fare la distinzione tra legge e massima, e anche principi. "Solo l'imperativo categorico va inteso come legge pratica, mentre tutti gli altri possono essere certo chiamati principi della volontà, ma non leggi" 25 . Senza la distinzione tra massima, come un principio soggettivo dell'agire, e la legge, come un principio oggettivo che contiene il fondamento secondo cui si deve agire, non si riesce a capire la possibilità dell'imperativo categorico.

Esiste un collegamento sintetico stabilito tra la volontà e la legge, ma questo collegamento è a priori giacché niente di empirico può fondare la moralità. Kant chiama questa proposizione sintetico-pratica a priori, la quale deve costituire l'oggetto di una critica analoga a quella della possibilità dei giudizi sintetici a priori ${ }^{26}$. Dunque, si può affermare che nella Fondazione Kant prepara il terreno della Critica della ragione pratica. Siamo arrivati a un momento decisivo del pensiero kantiano presente nella Fondazione. Lo si può precisare con la seguente domanda: come stabilire la formula dell'imperativo universale del dovere?

L'intenzione di Kant è esprimere, nella formula, il significato di "necessità pratica assoluta". Kant prende in considerazione la questione chiedendosi "se forse il semplice concetto di un imperativo categorico non ci fornisca anche la sua formula, la quale contiene la proposizione che, sola, può essere un imperativo categorico" 27 .

\footnotetext{
${ }^{21}$ Cf. KANT, 2009, p. 67.

22 Ibidem, p. 71.

${ }^{23}$ Cf. Ibidem, p. 73.

${ }^{24}$ Cf. GONNELLI, F. "Introduzione". In: ibidem, p. xv. Nel riguardo come sia possibile una tale proposizione, secondo Kant, è un problema la cui soluzione non sta più entro i confini della metafisica dei costumi (Cf. Ibidem, p. 125).

${ }^{25}$ Ibidem, p. 73. Per la distinzione tra legge e massima, Cf. Ibidem, p. 5, nota.

${ }^{26}$ Analogia alla Critica della pagion pura. In effetti è ciò che sarà studiato nella sezione terza della Fondazione e che costituirà l'oggetto della Critica della ragione pratica.

${ }^{27}$ Ibidem, p. 73.
} 
Secondo lui, l'imperativo, oltre alla legge, contiene solo la necessità che la massima sia conforme a questa legge, in modo tale che non resti altro che "l'universalità di una legge in generale come ciò a cui la massima dell'azione deve essere conforme" 28 .

E così, Kant arriva alla formula generale dell'imperativo categorico: "Agisci soltanto secondo quella massima per mezzo della quale puoi insieme volere che essa divenga una legge universale" ${ }^{29}$. Questa formula è il principio da cui possono essere dedotti tutti gli imperativi del dovere. Davanti all'universalità della legge si costituisce ciò che si chiama natura ${ }^{30}$. Con l'aiuto del concetto di "natura", Kant fa una precisazione dell'imperativo universale del dovere: "Agisci come se la massima della tua azione dovesse diventare per mezzo della tua volontà una legge universale della natura" ${ }^{\prime 3}$.

In sintesi, si può concludere che la formula fornita del concetto di dovere come adeguamento a una legge oggettiva e universale, esprime la "necessità pratica assoluta", cioè, la necessità dell'adeguamento della massima soggettiva alla forma di una legge come tale, che è sempre oggettiva e universale.

Dopo la formulazione della legge universale del dovere, Kant enumera alcuni doveri verso sé stessi e verso gli altri, doveri perfetti e doveri imperfetti. Come accennato precedentemente, Kant praticamente non offre esempi, però, secondo il parere di Filippo Gonnelli, che ha tradotto e fatto l'introduzione del libro Fondazione, Kant offre "quattro esempi di applicazione di questo imperativo, molto significativi per lo specifico orientamento della morale kantiana e anche molto noti, ma nei quali sono ben visibili problemi irrisolti" ${ }^{32}$. Ecco gli esempi forniti da Kant: il suicidio, la falsa promessa, il non coltivare i propri talenti e l'indifferenza verso i mali degli altri.

Suicidarsi a causa del tedio della vita o fare una promessa falsa per liberarsi da una difficoltà sono azioni in cui le massime non potrebbero diventare leggi universali della natura senza incorrere in contraddizioni. Rispetto al suicidio, che è un esempio del dovere perfetto verso se stessi, la massima secondo la quale, per amore di sé, togliersi la vita sembra un bene, non potrebbe diventare una legge universale perché contraddirebbe la stessa legge naturale. Secondo Kant, qui si può vedere subito che una natura la cui legge distruggere la vita per mezzo dello stesso sentire contraddirebbe se stessa e dunque non sussisterebbe come natura ${ }^{33}$.

Lo stesso ragionamento fa Kant in relazione alla falsa promessa. Con la promessa falsa, un esempio perfetto verso gli altri, la massima non potrebbe diventare una legge universale della natura perché la promessa smetterebbe d'essere una promessa. Non si potrebbe concepire un mondo in cui la legge fosse fare una promessa con l'intenzione di non eseguirla, perché se così fosse, la nozione di promessa perderebbe il suo senso.

Rispetto al terzo, ai talenti, c'è un esempio di dovere imperfetto verso sé stesso. Preferire abbandonarsi ai godimenti, piuttosto che sforzarsi di accrescere e migliorare le proprie fortunate disposizioni naturali, non può diventare una legge universale della natura perché un essere razionale vuole anche che tutte le sue facoltà vengano sviluppate.

\footnotetext{
${ }^{28}$ KANT, 2009, p. 75.

${ }^{29}$ Ibidem, p. 75.

${ }^{30} \mathrm{Nel}$ senso più generale, per Kant, natura è "l'esistenza delle cose in quanto è determinata secondo leggi universali" (Ibidem, p. 75).

${ }^{31}$ Ibidem, p. 75.

${ }^{32}$ GONNELLI, F. "Introduzione". In: Ibidem, p. xv.

${ }^{33}$ Cf. Ibidem, p. 77.
} 
La questione dell'indifferenza verso i mali degli altri è un esempio di dovere imperfetto verso gli altri. Per Kant, anche se il genere umano sussistesse secondo una tale massima, sarebbe tuttavia impossibile volere che un tale principio valesse come legge della natura, perché una volontà che optasse per questo principio contraddirebbe sé stessa, sarebbe priva di ogni speranza di aiuto presente nella comunità umana.

Perciò, in relazione a questi due ultimi esempi di doveri, che riguardano il non sviluppare i propri talenti e l'indifferenza ai mali degli altri, si può capire che sono forme di condotta di cui le massime non possono diventare leggi universali, perché, in verità, la pigrizia e l'egoismo sono forme d'agire immorali, il primo verso sé stessi e il secondo verso gli altri.

In sintesi, si può dire che, in relazione ai primi doveri, il suicidio e la promessa falsa, che sono esempi di doveri perfetti, la massima non può essere concepita senza contraddizione come legge universale della natura. Negli altri due esempi, che sono esempi di doveri imperfetti, la massima non può essere concepita come tale.

Si può concludere con Kant che "è necessario poter voler che una massima delle nostre azioni divenga una legge universale: questo è il canone in generale del giudizio morale sulla massima" 34 . E con questo principio, davanti ai quattro esempi, si avverte che: in alcuni azioni si può pensare la massima come legge universale della natura; in altri, è tuttavia impossibile volere che la loro massima venga elevata all'universalità perché in tale volontà si contraddirebbe.

In modo critico, secondo Kant, se soppesassimo tutto da un solo ed unico punto di vista, quello della ragione, troveremmo una contraddizione nella nostra volontà. Questa contraddizione, in verità, si deve al fatto che, oggettivamente parlando, c'è un certo principio che deve essere rispettato in modo necessario e oggettivo, in quanto legge universale, ma soggettivamente non dovrebbe valere in modo universale, bensì dovrebbe ammettere eccezioni. Tuttavia, ciò che definiamo come un'eccezione, nella maggior parte dei casi è un vantaggio della nostra inclinazione. Ciò che è comune a tutti, valido come principio della ragione pura è l'essere ragionevole. Però, l'uomo può agire in modo diverso. Ossia, in relazione alle inclinazioni della volontà, l'uomo può volere altre cose che non sono d'accordo con la ragione. Secondo Kant, con Dio non è così, perché Lui non può agire in modo differente. Per Dio non esiste legge morale, cioè, non esiste il "tu devi". E allora, negli uomini, la volontà "non buona" viene costretta dalla ragione, ciò che Kant chiama comandi o imperativi.

\section{Dall'intenzionalità alla prossimità}

L'alternativa che Levinas propone è quella di un ripensamento della condizione umana da un nuovo punto di partenza, vale a dire, dall'esteriorità. La base di ogni relazione etica è la relazione dell'uomo con l'esteriorità. Su questa nuova base tutta l'antropologia, la razionalità, la libertà, la volontà, l'autonomia, riceve un significato diverso. La posizione di Levinas consiste precisamente nel distinguere, forse contro l'umanesimo contemporaneo, la nuova via, al di là dell'immanenza dei concetti umanistici.

È necessario subito chiarire, però, che il progetto filosofico di Levinas non contempla la costruzione di un sistema morale. Anzi, lui tenta in qualche modo di decostruire i significati dei termini che compongono l'edificio della morale nella sua

\footnotetext{
${ }^{34}$ KANT, 2009, p. 81.
} 
concezione tradizionale per cercare il "senso" dell'etica ${ }^{35}$. L'espressione "cercare il senso" non va in alcun modo sottovalutata ${ }^{36}$.

Qual è il senso di un'etica? Non sarà sicuramente la formulazione di un doveressere al quale si arriva guidati da regole e direttive, ma un esercizio fenomenologico vale a dire, la constatazione di un fatto, la sua descrizione e la sua analisi, la descrizione della sua struttura formale. Ma non solo. L'etica per Levinas è fatta soprattutto di attenzione alle realtà umane, specialmente alla responsabilità di ogni essere libero.

Il soggetto kantiano rappresenta, come abbiamo già visto, quella unità formale, $\mathrm{o}$ coscienza razionale, nella quale si riconosce l'origine dei valori, delle norme e delle leggi. Per Levinas il soggetto libero kantiano è solo una modalità dell'unità logica. In etica, però, bisogna oltrepassare questo sistema. Perciò, in un primo momento, si cercherà di illustrare le peculiarità dell'etica levinassiana esponendo i concetti di esteriorità e sensibilità che hanno permesso a Levinas di rifiutare ogni tentativo di fondazione dell'etica sulla logica della ragione autosufficiente e idealista ${ }^{37}$.

In un secondo momento, saranno presentate le conseguenze di questo modo di pensare "altrimenti" l'esteriorità a la sensibilità. Vedremo che le riflessioni di Levinas portano a una conseguenza inevitabilmente etica: l'assoluta responsabilità per l'Altro, dove questa responsabilità per l'altro uomo si presenta come una nuova comprensione della soggettività.

\subsection{La sensibilità come apertura dell'intenzionalità}

Per Levinas esiste una realtà che sfugge a qualsiasi tentativo di universalizzazione che non può essere da me compresa e non può nemmeno unificarsi in un sistema perché si tratta, appunto, di una realtà assolutamente estranea e radicalmente separata dall'esperienza della conoscenza la quale culmina sempre nell' autocoscienza e nell'identificare ogni cosa all'essere.

Se si ammette l'esistenza di questa realtà, la comprensione della soggettività subisce delle trasformazioni. Si verifica ad esempio che il soggetto non può stabilire una legge morale universale in modo rigorosamente autonomo. Non lo può fare non solo perché porta nella sua coscienza innumerevoli significati e infiniti orizzonti, alcuni impliciti, altri oscuri, altri ancora dimenticati, ma soprattutto perché c'è di più oltre l'intellettualismo, sia esso razionalistico o sia empiristico ${ }^{38}$.

\footnotetext{
35 In un interessante dialogo con P. Nemo che ha dato poi origine al libro Etica $e$ infinito, Levinas è perentorio nell'affermare che non ha mai avuto l'intenzione di stabilire le regole per una morale (cf. LEVINAS, E. Etica e infinito, 90) in perfetta consonanza con il suo pensiero di un soggetto che non deve essere posto soltanto come correlato di un pensiero, ma di relazioni: atteggiamento proprio di un soggetto aperto a sensi molteplici.

${ }^{36}$ Sul tema del "senso" e dell'etica Levinas dedica un intero capitolo nel suo libro del 1972 Humanisme de l'autre homme dove arricchisce considerevolmente il significato del termine nel collegarlo con l'Altro: "Essi [gli altri] non sono né un significato culturale né un semplice dato. Sono senso in maniera primordiale (...), perché solo per mezzo loro un fenomeno come il significato entra, di sua natura, nell'essere" (LEVINAS, E. Umanesimo dell'altro uomo. Genova: Il Melangolo, 2009, p. 74).

${ }^{37}$ La discussione intorno alla ragione idealistica, intesa nel senso hegeliano, è uno dei temi centrali in Totalità e Infinito. Nel corso dell'opera infatti, Levinas ha cercato di mostrare che la ragione intesa come pensiero universale è antitetica all'etica proprio perché sopprimendo qualsiasi differenza e singolarità si pone come unità totalizzante. Per un approfondimento del rapporto con il pensiero hegeliano si rimanda anche al breve saggio Difficile Libertà. Saggio sul giudaismo.

${ }^{38}$ Sul tema della propensione all'altro si veda il testo Scoprire l'esistenza (Cf. LEVINAS, E. Scoprire l'esistenza con Husserl e Heidegger. Milano: Raffaello Cortina, 1998, p. 141-154) dove Levinas elabora una fenomenologia dell'intenzionalità riprendendo l'affermazione di Husserl al paragrafo 20 delle Meditazioni: "Questo intendere-oltre-di-sé che è in ogni coscienza, deve essere considerato un momento essenziale" (HUSSERL, E. Meditazioni cartesiane. Bergamo: Bompiani, 2002, p. 75). In Umanesimo
} 
Questo “oltre", questo “di più" o "alterità" è esteriorità: luogo dell'etica. L'idea di esteriorità è fondamentale per la comprensione di eteronomia nel pensiero di Levinas: di fronte alle cose non esiste solo la presenza della ragione, altre realtà sono a esse correlate, inclusa l'etica; perciò, ogni relazione è compromessa anche da questa presenza esteriore, non integrata nel mondo del soggetto.

È vero che 1" "io" nell'appercezione trascendentale porta tutto all'interno, concentra tutto, cerca di riflettere in sé ogni realtà. Ma è pur vero che l'io sperimenta anche il limite dell'appercezione trascendentale quando viene sconvolto da qualcos'altro non inserito nell'unità dell'Uno; quando è sorpreso dalla visita inaspettata dell'"Altro", "come se l'intempestivo venisse a sconvolgere le concordanze della rappresentazione. Come se una estranea debolezza scuotesse di brividi e facesse vacillare la presenza o l' essere in atto" 39 .

La venuta dell'Altro tocca la sensibilità dell'Uno: l'uno è sensibile all'altro; “è il per l'altro di un essere che si dis-tacca, senza fare di sé il contemporaneo dell' 'altro', senza potersi mettere accanto a lui in una sintesi esponibile come un tema" ${ }^{40}$. Questo perché Altri non sono le espressioni che usiamo per dare significato alle cose, per esprimere l'essere, "sono senso in maniera primordiale, perché ne danno uno all'espressione stessa, perché solo per mezzo loro un fenomeno come il significato entra, di sua natura, nell'essere" 41 tramite la sensibilità. Il termine "espressione" rimanda anche a un altro termine, quello di volto.

Il volto è espressivo, parla senza parola, è ciò che vi è di più nudo, di più scoperto, e dunque si riferisce a tutto quello che è relativo all'alterità. Il senso del volto, come il volto di una persona, è ciò che c'è di più spogliato, più esposto e vulnerabile. Non possiede forma né contenuto culturale. Ma il volto è anche ciò che resiste ad ogni dissoluzione della sua alterità attraverso una rappresentazione o un concetto. Diverso dal fenomeno che è sempre, comunque lo si intenda, immagine, tematizzazione ${ }^{42}$ il volto semplicemente parla ${ }^{43}$, fa il suo ingresso, viene, arriva, è visitazione.

Il problema principale che Levinas affronta è proprio questo: come è possibile che il volto non sia semplicemente una rappresentazione? Perché l'Altro non può essere preso intenzionalmente? E risponde: perché si tratta di una presenza al di là dell'essere, in altre parole, non è un contenuto, modo per cui la coscienza intenzionale non lo può osservare.

Ma bisogna chiarire meglio il significato di al di là. Se la straordinaria esperienza dell'ingresso e della visitazione conserva il suo significato, allora sarà questo: che al di là non è un "altro mondo" dietro il mondo. L'al di là è precisamente l'al di là del mondo, vale a dire, al di là di ogni possibile svelamento e di ogni possibile identificazione con il mondo ${ }^{44}$.

La coscienza, in effetti, ha a che fare con questo mondo. Essa si caratterizza come un godere del mondo e un abitare in esso. Noi crediamo di controllare tutto intorno a noi, di conoscere bene e di decidere liberamente dove posare le cose all'interno del nostro mondo in modo da sentirci sicuri e non essere sorpresi dallo

dell'altro uomo invece stabilisce le condizioni di tale orientamento: "Esso non può essere posto altrimenti che come un movimento che va fuori dell'identico, verso un altro che è assolutamente Altro" (LEVINAS, 2009, p. 65-66).

${ }^{39}$ LEVINAS, 2009, p. 25.

${ }^{40}$ Ibidem, p. 24.

${ }^{41}$ Ibidem, p. 74.

${ }^{42}$ Cf. Ibidem, p. 75.

43 Spiega Levinas: "Parlare è, prima di ogni altra cosa, questo modo di venir fuori da dietro la propria apparenza, da dietro la propria forma, un'apertura nell'apertura" (Ibidem, p. 75-76).

${ }^{44}$ Cf. Ibidem, p. 88. 
sconosciuto. Ordiniamo tutto, niente sfugge allo sguardo della nostra intenzionalità. Nonostante questo, scrive Levinas: "Il viso entra nel nostro mondo avanzando da una sfera assolutamente estranea, ossia, precisamente, da un as-soluto, che è poi il nome stesso della estraneità più profonda. La significanza del viso nella sua astrattezza, è, nel senso letterale del termine, straordinaria, fuori di ogni ordine, di ogni mondo" $"$.

A proposito della visitazione del volto, Derrida nel suo libro Addio a Emmanuel Levinas, afferma che l'ospitalità è l'etica stessa, il suo principio, se è vero che ethos rimanda appunto all'abito, all'abituale, all'abitudine, e quindi anche all'abitare: accogliere l'altro che viene, farsi abitare dall'altro sarebbe l'unico imperativo di un'etica che di fatto si confronta con il problema dell'alterità: apertura della porta di casa come segno di apertura dell'intenzionalità alla sensibilità. Commenta Derrida: "non c'è intenzionalità prima e senza quell'accoglienza del volto che si chiama ospitalità (...). Viceversa, non si capirebbe nulla di ospitalità senza illuminarla con una fenomenologia dell'intenzionalità, una fenomenologia che rinuncia addirittura, laddove è necessario, alla tematizzazione" 46 .

Il volto, perciò, non è tanto qualcosa che si guarda, quanto la voce che si ascolta e a cui si risponde. La sua parola non viene colta, ma piuttosto accolta. Accogliere l'Altro significa, quindi, ricevere una parola che consente di condividere un mondo e una casa. Questo è importantissimo! Vuol dire che il senso dell'etica viene condiviso. Il soggetto non può trarre da sé i principi etici.

È in questo senso che la soggettività è sensibilità per "altri”, cioè, riceve qualcosa di estraneo, di straniero. La coscienza di sé è anche e comunque coscienza dell'altro, sensibilità o godimento e allo stesso tempo responsabilità-per-l'altro fino alla sostituzione $^{47}$. Solo a partire dall'esposizione della soggettività sensibile è possibile cogliere il senso dell' etica come dono che arriva, come visita. Di fatto, la sensibilità non viene considerata come semplice materia puramente data sulla quale i sensi si esercitano e il pensiero la coglie, ma luogo stesso dell'accoglienza: il ricevere dall'esterno sembra la caratteristica più esatta della sensibilità.

La soggettività come sensibilità è senz'altro autonoma, ma di una autonomia interrogata dall'Altro. In tal modo si può affermare che la vita morale in quanto tale è costituita dal senso dell'Altro che investe la soggettività ed è perciò completamente eteronomia. Scoprire l'eteronomia è scoprire l'orientamento in senso unico ${ }^{48}$ della coscienza. In senso unico perché l'Altro è metafisico per eccellenza: non è possibile la reciprocità Io-Altro.

Sotto un aspetto più positivo tuttavia, l'eteronomia che all'inizio sembra limitare la libertà, è quello che la rende valida perché le dà un confine: fino alla resistenza etica. "L' 'assolutamente altro', scrive Levinas, non si riflette nella coscienza. Le resiste a tal punto che neanche la sua resistenza si converte in contenuto di coscienza"49.

Levinas esplicita l'idea di resistenza fornendoci alcuni esempi che sono "a portata di mano": la resistenza all'assassinio. Se la resistenza all'assassinio fosse reale e

\footnotetext{
${ }^{45}$ LEVINAS, 2009, p. 76.

${ }^{46}$ DERRIDA, J. Addio a Emmanuel Levinas. Milano: Jaca Book, 2011, p. 114.

${ }^{47}$ Il termine sostituzione appare al capitolo IV di Altrimenti che essere e rappresenta un'elaborazione di una conferenza tenuta da Levinas il 30 novembre del 1967 a Bruxelles, e poi pubblicata nella "Revue philosophique de Louvain" nell'ottobre del 1968. Siccome il soggetto etico sopporta tutto, si fa dono, si svuota, offre sé stesso per l'altro, la sostituzione è il tratto che meglio descrive la soggettività etica. Sostituirsi è "come avere-l'altro-nella-propria-pelle" (LEVINAS, E. Altrimenti che essere o al di là dell'essenza. Como: Jaca Book, 2011, p. 143).

${ }^{48} \mathrm{Si}$ capisce senso unico anche nel movimento dell'uno per l'altro, cioè, quando entra il "Terzo", colui che è prossimo mio e dell'altro, il senso cambia ed entra la giustizia, la reciprocità, la politica.

${ }^{49}$ LEVINAS, 2009, p. 77.
} 
non etica avremmo accesso a una realtà o molto debole o molto forte. Un altro esempio che ci viene dato riguarda la libertà. Se la libertà fosse messa in questione da un'altra libertà, allora ci sarebbe una tensione tra due volontà autonome e, al limite, una lotta. Ma invece, un essere libero solo si riconosce tale in presenza d'Altri. Allora, la sua libertà si rivela illegittima e ingiusta. Ora, il fatto di sapersi ingiusti e colpevoli "non è qualcosa che si aggiunge a una coscienza spontanea e libera" ${ }^{90}$.

Come si vede, non si tratta affatto di una lotta tra due libertà, o una lotta di potere. Semmai è una lotta nella quale il vincitore esce ferito, non perché i suoi poteri siano troppo deboli, ma perché è lasciato libero ${ }^{51}$. Il soggetto levinassiano non è libero di scegliere di compiere il dovere. Esso va ben oltre la sua previa decisione: s'impone già da un tempo immemorabile.

Infine, si può affermare che per Levinas l'eteronomia è quello spazio morale di resistenza alle varie forme di totalizzazione e violenza. Commenta Gates: "Ciò significa che la resistenza morale del rapporto faccia-a-faccia ci espone alla nostra responsabilità senza costringerci a rinunciare alla nostra capacità di azione" ${ }^{\circ 2}$.

\subsection{La chiamata alla responsabilità}

Come abbiamo visto è la stessa sensibilità che ci apre all'etica. Grazie alla sensibilità il soggetto può veramente dire: c'è "Altro oltre" il mio mondo. Una volta detto questo bisogna vedere che tipo di rapporto viene stabilito. Abbiamo già visto che nel rapporto Io-Altro c'è un'evasione dal piano intenzionale: l'Altro resta sempre altro nonostante ogni tentativo di assimilazione conoscitiva.

Non si tratta di un rapporto di reciprocità temporale, perché l'Io non è mai contemporaneo dell'Altro ${ }^{53}$, nemmeno di distanza spaziale: l'altro è sempre prossimo. Questo è il senso della prossimità: la non coincidenza spazio-temporale dell'io rispetto all'etica. Ben inteso, non si tratta della presenza oggettiva di un "tu" che venga dopo, o magari prima, dell'esperienza dell'essere, ma dall'impossibilità di una sincronia o reversibilità: la prossimità del volto impone una relazione totalmente di tipo etico.

Una relazione etica implica, secondo Levinas, l'obbedienza. Essa viene intesa in un modo del tutto originale. È una obbedienza di tipo metafisico, cioè precede l'ascolto di qualunque comando o imperativo che non sia quello della voce dell'Altro che chiama in giudizio prima che uno si riconosca un essere libero ${ }^{54}$. Essere in rapporto etico è poter dire con tutta la franchezza: c'è l'Altro e gli debbo obbedienza, gli debbo tutto.

Il discorso sull'obbedienza può suonare scandaloso per la coscienza dell'uomo contemporaneo. Ma lo scandalo forse verrebbe minimizzato se si comprendesse che l'unico obbligo dato dal "di fuori" al quale il soggetto "deve" sottostare è questa

\footnotetext{
${ }^{50}$ LEVINAS, 1998, p. 193.

51 Ricordiamo l'episodio misterioso del Libro della Genesi 32,23-33 dove Giacobbe lotta contro uno sconosciuto. Nell'esperienza di Giacobbe, l'Altro si presenta con l'ambiguità del prossimo, provocante ed inquietante. La lotta di Giacobbe con l'innominato diventa paradigma della relazione con Altri (Cf. D'ANTUONO, E.; KAJON, I.; SINDONI, P.R. Giacobbe e l'angelo: figure ebraiche alle radici della modernità europea. Roma: Lithos, 2012).

52 GATES, D.C. "The Fact of Reason and the Face of the Other: Autonomy, Constraint, and Rational Agency in Kant and Levinas". The Southern Journal of Philosophy. v.40, n.4, 2002, p. 493. (traduzione nostra).

${ }^{53}$ Per una conoscenza più approfondita della meditazione di Levinas sul tempo si rimanda a $I l$ tempo $e$ l'altro, frutto di quattro conferenze tenute dall'autore nel 1946-47 all'inizio dello sviluppo del suo pensiero. Notassi soltanto che secondo Levinas il tempo non è una categoria del pensiero ma un modo specifico di relazione faccia-a-faccia con l'altro (cf. LEVINAS, E. Il tempo e l'altro. Genova: Il Melangolo, 1993, p. 11).

${ }^{54}$ Cf. LEVINAS, 2009, p. 145.
} 
chiamata a non anteporre nulla all'Altro, nemmeno le giuste "massime universali". La coscienza morale è "la coscienza del privilegio di Altri rispetto a me"

Il volto dunque è anche manifestazione di una parola, di un precetto, che direttamente chiama, interpella. È una parola di interdizione: "non uccidermi”, che vieta ogni tentativo di sopprimere l'alterità dell'Altro. La risposta a questo appello che l'Altro mi rivolge diviene così un modo per scoprire la mia stessa identità, in quanto soggetto non chiuso in sé, ma aperto all' Altro, per-l'Altro, responsabile dell'altro.

La parola "responsabilità", spiega Levinas, non è che un modo di esprimere questo: che l'io è responsabile di altri, risponde di altri, e sostanzialmente risponde prima ancora volerlo fare. Il paradosso della responsabilità, è che essa non è il risultato di un atto qualsiasi commesso o di un impegno della propria volontà. È come se l'io fosse responsabile prima di volerlo essere, prima d'aver commesso qualsiasi cosa, come un a priori e, di conseguenza, come se non fosse "libero" di liberarsi di tale responsabilità, infine, come si dovesse comportare da ostaggio per l'altro ${ }^{56}$, o ancora come se dovesse comportare con la dignità del figlio maggiore di fronte ai fratelli o con l'onore di essere stato eletto. E tuttavia, la responsabilità per l'altro non produce dipendenti: essere eletti non significa schiavitù.

È in virtù di questa chiamata, primogenitura o elezione che liberamente uno può farsi ostaggio per l'altro. Questo passaggio è importante. Bisogna essere cauti. Vuol dire che l'eteronomia non significa essere dipendenti da una legge che viene dal di fuori della volontà, come è stata sempre intesa; significa piuttosto la liberazione dell'io dalle trame dei propri stati di coscienza. In termini più semplici, eteronomia è una situazione dove la coscienza viene destituita dal suo posto di onore.

Levinas però va oltre passando dalla nozione di coscienza destituita a quella di "cattiva coscienza" 57 : la vera eteronomia nel pensiero levinassiano incomincia quando la coscienza cessa di essere coscienza obbediente e diventa cattiva coscienza. Secondo lui, l'intera tradizione occidentale si è sempre identificata con la ricerca di unità del pensiero nell'essere, perciò non le causa nessuna stranezza il fatto di aver assistito e anche subito ogni sorta di esclusione e di distruzione della differenza considerata disintegrata dall'Essere e quindi, destinata a sparire.

Secondo questa lettura della tradizione, l'uomo occidentale si è sempre scoperto buono e quindi naturalmente inclinato a ripetersi e a rifiutare il diverso. Una coscienza naturalmente buona è ritenuta tale perché è una coscienza a misura mia, cioè, la volontà accoglie il giudizio e lo fa suo. In questo caso l'eteronomia viene assorbita nell'autonomia ${ }^{58}$.

Qui l'importanza della parola "differenza" che Levinas riprende forse più dal mondo ebraico che da quello greco ${ }^{59}$. Di fatto, Levinas non intende la differenza in

\footnotetext{
${ }^{55}$ LEVINAS, 1998, p. 199.

${ }^{56}$ La nozione di ostaggio viene ripresa più volte da Levinas ed è sviluppata in modo complesso nelle sue opere principali. Ma in un dialogo con B. Casper che lo intervistava l'ha sintetizzata così: "E l'essere ostaggio è forse solo un nome più forte per dire l'amore" (LEVINAS, E.; CASPER, B. In ostaggio per l'altro. Pisa: ETS, 2012, p. 22).

${ }^{57} \mathrm{Da}$ non confondere con l'interpretazione nietzschiana. Come è noto, per Nietzsche la cattiva coscienza è la malattia dell'uomo dalla quale bisogna guarire per poter diventare uomo nuovo, libero da ogni senso di colpa (Cf. NIETZSCHE, F. Genealogia della morale. Milano. Milano: Adelphi, 1984, p. 78).

${ }^{58}$ Cf. LEVINAS, 1998, p. 202.

${ }^{59}$ Una delle caratteristiche del pensiero giudaico è la continua reinterpretazione della figura ontologica dell'ebreo errante chiamato a "uscir fuori di sé" e inevitabilmente incontrare l'esteriorità, la differenza, pur sempre soltanto nella traccia dell'Altro. Abramo ha insegnato che un'esistenza sensata e/o sensibile non può farne a meno di questo incontro, perché affinché l'esteriorità, la differenza, possa entrare in rapporto con l'uomo è necessario seguire le tracce, cioè, che si produca un'epifania, una rivelazione (Cf. CHALIER, C. Judaisme et alterite. Lagrasse: Verdier, 1982, p. 281).
} 
quanto differenza ontologica come ha fatto Heidegger, ma piuttosto come differenza etica, cioè, come scissione tra il Medesimo e l'Altro. La differenza sarebbe quel "movimento che va fuori dell'identico, verso un Altro che è assolutamente altro"60.

La tendenza a considerarsi buono è evidente dal momento che si trova sempre delle ragioni che giustifichino quello che si fa. È difficile cogliersi cattivi. E quando eventualmente lo si riconosce è sempre come un fatto isolato e in ritardo rispetto agli eventi. Ma in verità, uno può sempre sperimentarsi naturalmente cattivo ogni volta che prova a passare con indifferenza sotto lo sguardo altrui senza riuscirvi, cioè, non senza sentirsi colpevole e responsabile. Ma perché mi sento così? Levinas formula tre ipotesi: a) perché nella visitazione del volto si trova un comandamento; b) perché allo stesso tempo, tuttavia, il volto di altri è spoglio, nudo, povero; c) e io, chiunque io sia, proprio in quanto "prima persona", sono colui che ha sufficienti risorse per rispondere all'appello ${ }^{61}$.

L'Altro viene a me, nudo, spogliato della forma, e mi si propone come "senso". Allora regredisco al di là della mia buona coscienza: non c'è niente che mi giustifichi di fronte ad un volto che si presenta in questo modo. Di fronte a lui mi trovo cattivo e responsabile. Quindi la vera origine della mia coscienza è un volto che mi viene incontro. In altre parole, l'esperienza del volto dell'Altro è la breccia nella mia coscienza che mi permette di pensare moralmente e agire eticamente.

Se per Kant la morale dev'essere frutto dell'attività della ragione, attività propria di un soggetto autonomo, per Levinas invece, il primo dato di una coscienza morale non è un'attività della ragione ${ }^{62}$ ma è l'obbedienza nel senso di pazienza, inclinazione, in ultima analisi, di desiderio dell' Altro, quel 'trasporto puro, l'orientamento assoluto, il senso"63.

Desiderio senza soddisfazione che, appunto, mantiene la differenza propria dell'alterità e l'esteriorità dell'Altro: non per niente il vocabolo "desiderio" rimanda etimologicamente ai sidera, alle "stelle", all'Infinito. Questo desiderio o "questa singolare obbedienza all'ordine di arrendersi, senza comprensione dell'ordine, questa obbedienza anteriore alla rappresentazione, questa fedeltà prima di ogni giuramento, questa responsabilità preliminare all'impegno, è precisamente l'altro-nel-medesimo, ispirazione e profetismo, l'accadere dell'Infinito" 64 .

Infinito, afferma Levinas, è nell' ambivalenza del desiderio ${ }^{65}$. Il desiderio accade anche nel soggetto ma proviene in realtà dall'Altro, da fuori. Non diventa mai bisogno o possesso perché sempre separati. Essendo desiderio, mantiene accesa la fiamma del pensiero perché consiste, paradossalmente, nel pensare più di ciò che è pensato, conservandolo, così, nella sua dismisura, rispetto al pensiero. Levinas intravede così la possibilità di attenuare ogni pretesa di dominio della soggettività: è l'altro a concedere la possibilità di conoscere senza diventare conoscenza. “(...) l'Infinito non è un correlativo, come se tale idea fosse ancora un'intenzionalità, che si riempie nel suo oggetto. Il prodigio dell'Infinito nel finito di un'idea sta nello sconvolgimento dell'intenzionalità, nello sconvolgimento di quell'appetito di luce che è l'intenzionalità'66.

\footnotetext{
${ }^{60}$ LEVINAS, 2009, p. 65. diritto di serbare nulla" (Ibidem, p. 72).

${ }^{62}$ Cf. LEVINAS, E. Di Dio che viene all'idea, p. 199.

${ }^{63}$ LEVINAS, 2009, p. 73.

${ }^{64}$ LEVINAS, 2011, p. 189.

${ }^{65}$ Cf. LEVINAS, 2009, p. 79.

${ }^{66}$ Ibidem, p. 79.
}

61 "La relazione con gli Altri mi rimette in discussione, mi svuota di me stesso e non finisce mai di svuotarmi, scoprendo in me sempre nuove risorse. Non sapevo di essere tanto ricco, ma non ho più il 
Non si tratta però di una violenza fatta allo spirito conoscitivo ${ }^{67}$, nemmeno di comandamenti che vengono imposti alla volontà. L'autonomia viene preservata perché nella sua ipseità l'io è totalmente indipendente dall'Altro: in effetti, a "casa propria" l'io possiede sé stesso integralmente. E Altro? In questo piano, Altro si trova in condizione di straniero. E quindi, non rappresenta un limite per la libertà. Semmai è una proposta di apertura del mondo del soggetto che diventa capace di conoscere che c'è dell'altro, che c'è qualcos'altro al di là delle sue certezze, sicurezze, valore. Altro non mi limita, né aliena ${ }^{68}$, ma piuttosto mi chiama ed elegge e comanda. Comanda tuttavia in un modo del tutto extraordinario ${ }^{69}$, con la sua prossimità: "L'altro uomo comanda con il suo viso" ${ }^{, 70}$. La presenza di Altri, dunque, non annulla la ragione, tanto meno la libertà. Se così fosse, la concezione levinassiana di etica sarebbe la più irrazionale e acritica della storia. Il nodo della questione sta nel fatto che Altri si trova al di là, è esteriorità assoluta rispetto alla coscienza costituente. Solo così, dopo la filosofia kantiana, l'eteronomia viene riabilitata in un senso preciso, ossia, in quanto significa quella "resistenza etica" che è la propria presenza d'Altri.

Nel commisurarsi con l'Altro la libertà nel suo stesso esercizio si scopre usurpatrice. Perché in fondo è l'Altro che le dona il senso. Se l'uomo è responsabile di qualcosa, è proprio di questo senso che gli è stato donato. Quindi, si può dire, che l'uomo nasce responsabile prima ancora di essere libero: egli si trova originariamente assegnato alla responsabilità, prima di ogni eventuale accettazione o rifiuto. $\grave{E}$ precisamente un essere libero che è responsabile: esso si trova dunque già impegnato per sua propria libertà.

La soggettività, dunque, non è un per sé ma uno per l'altro. Questo è il senso e anche il peso della responsabilità. Così viene resa completa la tesi principale di Levinas: la relazione di prossimità etica si fonda in quella responsabilità alla quale sono chiamato attraverso l'Altro.

\section{La fonte della moralità: tra Kant e Levinas}

Sicuramente il confronto tra Levinas e Kant risulta molto proficuo, se non altro perché il primo considera il secondo come colui che ha reso la fonte etica la vera fonte del senso. I riferimenti a Kant offrono spunti che suscitano la riflessione di Levinas.

In una nota all'introduzione di Altrimenti che essere, Silvano Petrosino rileva il fatto che la "critica del linguaggio ontologico, delle possibilità di questo linguaggio, e apertura alla riflessione etica sembrano affiancare immediatamente la riflessione kantiana al pensiero di Levinas"71. Ma subito dopo, però, avverte il rischio di questo avvicinamento. Lo stesso fa Gates, il quale sostiene la compatibilità dei due progetti non senza una revisione della nozione levinassiana di eteronomia e della nozione kantiana di

\footnotetext{
${ }^{67}$ Levinas non ha mai rifiutato di porsi la domanda: "Ma è proprio necessario rinunziare al sapere a ai significati per ritrovare il senso? "(LEVINAS, 2009, p. 65).

68 A differenza di come pensava Jean Paul Sartre, il Volto dell'altro non fa del soggetto un suo oggetto, ma annuncia l'umano, cioè, fonda l'etica che quello che di più umano possa esserci. Scrive Levinas: "In Sartre lo sguardo dell'altro, 'le regard de l'autre', contiene sempre un giudizio e una condanna. Scaturisce da una certa qual negatività che sussiste sempre tra me e l'altro. Ciò si fonda sulla vecchia ontologia del per-sé e dell'in-sé (...). Secondo me, invece: nell'ontologia vi è un'irruzione dell'umano" (LEVINAS; CASPER, 2012, p. 22).

${ }^{69}$ Straordinarietà nel senso anche di esteriorità ovvero eteronomia: "La significanza del viso, nella sua astrattezza, è, nel senso letterario del termine, straordinaria, fuori di ogni ordine, di ogni mondo (...) viso che "sfonda" l'ordine del mondo, e sullo sconvolgimento della coscienza che corrisponde a questa 'astrazione"” (LEVINAS, 2009, p. 76).

${ }^{70}$ Ibidem, p. 27.

${ }^{71}$ PETROSINO, S. “Introduzione”. In: LEVINAS, 2001, p. xxv.
} 
autonomia $^{72}$. Si cercherà di confrontare i due autori su ciò che riguarda la fonte della moralità a partire dai seguenti punti: l'autonomia e l'eteronomia, la ragione e la sensibilità seguendo anche le riflessioni di Catherine Chalier ${ }^{73}$.

\section{1. l'autonomia e l'eteronomia}

Il punto di partenza di Kant è la finitudine del soggetto. Come già abbiamo visto, da una parte esiste la "massima" che è un principio soggettivo dell'agire; dall'altra, questo principio si collega alla "legge" pratica come principio oggettivo, valido per ogni essere razionale, come imperativo ${ }^{74}$, in cui si trova la fonte del comandamento.

La domanda principale di Kant non è solo "come è possibile" l'imperativo, ma anche, come è possibile il dovere. In modo generale, si è in grado di dire che se il dovere è un concetto che deve possedere significato e reale legislatività per le nostre azioni, allora questa legislatività dev'essere espressa solo in imperativi categorici e mai ipotetici. Negli ipotetici, ciò che è desiderabile si trova presente nei mezzi e dipende della condizione: "se vuole questo, fa questo". Il senso è fuori dall'azione. Al contrario, nell'imperativo categorico, il dovere non dipende dalla condizione, il senso si trova nell'azione in sé. Perciò, secondo Kant, l'imperativo categorico è chiamato imperativo della moralità e la proposizione dell'imperativo categorico viene chiamata sinteticopratica a priori.

Ciò che viene dalla particolarità naturale dell'umanità può darci una "massima", ma non una "legge", e dunque, ha la necessità di un principio che sia oggettivo ed universale come formula del dovere. Dal punto di vista della filosofia "pratica", secondo il parere di Kant, questa riflessione tocca il proprio senso della filosofia. Se nella prima sezione della Fondazione Kant era arrivato all'affermazione che la comune ragione ha bisogno di uscire dal proprio ambito e proiettarsi nel campo della filosofia pratica per capire il suo principio di azione. Nella seconda sezione, arriva a una situazione difficile della filosofia: "aggrapparsi in cielo" o "appoggiarsi sulla terra?"75.

Senza dubbio, possiamo dire che Kant rimane fedele al suo principio del dovere. Perché, secondo lui, nulla è da attendersi dalla inclinazione dell'uomo, tutto invece dal supremo potere della legge e dal rispetto che le è dovuto. Dunque, in modo ancor più radicale dice che tutto ciò che è empirico, "non solo è completamente inutile, ma persino altamente dannoso per la purezza dei costumi""

Partendo dal principio della ragione pura - che l'uomo è un essere ragionevole e che ha la facoltà di agire secondo la rappresentazione delle leggi - allora si può concludere che il compito della filosofia è anche quello di custodire le leggi, aiutare l'uomo ad agire secondo il principio della moralità. Senza questa chiarezza, come dice Kant, l'uomo sarebbe condannato al disprezzo di sé e all'abominio interiore.

Levinas parte ugualmente dalla modernità, ma con sfumature molto diverse, ad esempio, lui fa una rilettura di Cartesio e scopre, nell'analisi dell'infinito che abita questa finitudine del soggetto, un'apertura all'esteriorità. E questa sarà una grande "porta aperta" per capire anche il senso della filosofia morale.

Mentre Kant cerca di fare un'analisi della "volontà buona" del soggetto morale, difendendo la volontà autonoma, Levinas parte dall'eteronomia morale, che significa

\footnotetext{
${ }^{72}$ Cf. GATES, 2002, p. 493.

${ }^{73}$ Nella sua opera Pour une morale au-delà du savoir. Traduzione nostra.

${ }^{74}$ KANT, 2009, p. 75 (nota).

${ }^{75}$ Cf. Ibidem, p. 85.

${ }^{76}$ Ibidem, p. 85.
} 
anche l'imperativo dell'esteriorità. Secondo Kant, non si possono dedurre gli imperativi della moralità degli oggetti o delle situazioni empiriche ossia di una fonte eteronoma ${ }^{77}$.

È chiaro in Kant che l'autonomia della volontà è fonte per se stessa, ossia, per la legge morale. In questo senso, l'eteronomia sarebbe strana, perché implicherebbe fondare la morale su leggi esterne, su imperativi ipotetici, o anche su sé stesso, come è il caso del suicidio e della promessa falsa che "contraddirebbe se stessa e dunque non sussisterebbe come natura" 78 .

Però, qui sorge una domanda: c'è un interesse puro, al di là dell'egoismo? Secondo Levinas, la morale non comincia con l'interesse, ma con il disinteressamento. Questo è il grande dibattito dell'opera Altrimenti che essere, nella quale Levinas introduce nell'essere un momento di pura gratuità ${ }^{79}$. Lui vuole passare all'altro dell'essere $^{80}$ e questo indica un movimento che pervade tutta la sua opera, l'essere l'uno-per-l'altro. Secondo lui, "a rigore altri è 'fine', io sono ostaggio, responsabilità e sostituzione sopportante il mondo nella passività della convocazione che arriva fino alla persecuzione accusatrice, indeclinabile" 81 .

È vero che anche Kant pensa un'antropologia in cui l'umanità non può essere mai pensata come mezzo, ma come fine in sé stessa. Ciò che Levinas critica è l'antiumanesimo moderno, ossia, l'umanesimo che non è sufficientemente umano ${ }^{82}$. A nostro modo di capire pensare l'etica in senso levinassiano, significa anche cambiare il modo moderno di ragionare, caratterizzato dall'attacamento al soggetto e alla centralità della libertà. In questo senso, molto vicino alla questione sull'autonomia-eteronomia, si trova un altro punto importante divergente tra Levinas e Kant che è la libertà e la responsabilità. Per Kant, se l'uomo non fosse libero non ci sarebbe la moralità. La libertà, dunque, viene prima ed è il fondamento dal quale fondatrice da cui si deduce la responsabilità ${ }^{83}$. Secondo il pensiero levinassiano, il soggetto è chiamato ad una responsabilità come un' "assegnazione dell'uno attraverso l'altro" ${ }^{84}$. E così si capisce perché per Levinas la responsabilità viene prima della libertà.

\subsection{La ragione e la sensibilità}

Diversamente da Kant, l'affetto ed i moventi sensibili non costituiscono, per Levinas, un problema. Al contrario, la sensibilità è etica. Con Levinas, il soggetto morale non è autonomo e, per questo, bisogna pensare la sensibilità come esposizione del soggetto all'altro, nella sua vulnerabilità, nelle sue sofferenze, e nella sua passività ${ }^{85}$.

Con Kant, c'è la "costrizione" della ragione sulla volontà che implica il dovere di trattare l'umanità nella sua persona. Questo funziona tra esseri finiti ed anche

\footnotetext{
${ }^{77}$ Cf. CHALIER, C. Pour une morale au-delà du savoir. Kant et Levinas. Paris: Albin Michel, 1998, p. 75 .

${ }^{78}$ KANT, 2009, p. 77.

${ }^{79}$ Cf. CHALIER, 1998, p. 80.

${ }^{80}$ Cf. LEVINAS, E. Altrimenti che essere, 6.

${ }^{81}$ LEVINAS, 2011, p. 161.

${ }^{82}$ Così come appare nel contesto dell'opera Umanesimo di altro uomo.

${ }^{83}$ Cf. CHALIER, 1998, p. 14. La questione sulla libertà è molto complessa in Kant. L'intento qui è solo di accennare la problematica.

${ }^{84}$ LEVINAS, 2011, p. 172. Questa "assegnazione" (assignation) è uno dei termini più caratteristici del lessico levinassiano. Secondo S. Petrosino, nelle note di traduzione all'opera Altrimenti che essere, xxxii, il verbo assigner ha due significati: 1) assegnare, fissare un compito; 2) citare, convocare. Perciò, da una parte, assignation sottolinea la "passività" di una chiamata; dall'altra parte, l'attività di un compito da assolvere, di una responsabilità.

${ }^{85}$ In riferimento a questo punto, si invita a leggere soprattutto il capitolo terzo di Altrimenti che essere, di Emmanuel Levinas.
} 
ragionevoli. Secondo Chalier, sembra che il "rispetto" per gli altri non è un rispetto per l'autorità dell'altro, ma per un alter ego, ossia, per un altro essere razionale come io, e non come alterità ${ }^{86}$.

In questa riflessione sull'alter ego si capisce la critica di Levinas a Kant che si trova nel titolo del libro di Chalier: Pour une morale au-delà du savoir. Kant insiste nel privilegiare una morale fondata sul sapere. Uscire da questa struttura significa per Levinas considerare la sensibilità nel senso etico e non epistemologico come ha fatto Kant.

Per Levinas, il soggetto emerge nella sua unicità, dell'elezione senza scelta ${ }^{87}$. E così si può affermare con Chalier: "a differenza di Kant, Levinas non pensa che il problema sia morale e libero grazie alla sua autonomia, ma a causa di questa elezione" ${ }^{\prime 88}$. Differentemente della modernità che ha privilegiato il soggetto, Levinas parla di un" "identità di pura elezione", chiamata anche come "identità pre-originale, anarchica, più antica di ogni inizio; non coscienza di sé che si raggiunge nel presente, ma esposizione estrema alla convocazione attraverso Altri, già compiuta prima della coscienza e della libertà, convocazione entrata in me per effrazione - vale a dire senza che 'appaia' e che parla nel Dire del convocato" 89.

Questa anteriorità dell'elezione è descritta da Levinas nell'ordine della "Bontà del Bene" e anche del "Bene prima dell'essere. Diacronia: differenza insormontabile tra il Bene e me, senza simultaneità dei termini divisi" ${ }^{90}$. In verità, c'è in Levinas una differenza tra i termini della relazione, ma nella non-in-differenza. Da qui viene anche l'interpretazione per capire l'asimmetria del rapporto etico in Levinas.

\section{Conclusione}

Seguendo Chalier si può dire che Kant e Levinas hanno in comune la ragione pratica che oltrepassa i limiti della ragione speculativa. E così, Kant e Levinas si sforzano di ottenere un' etica che non si basa sull'ontologia ${ }^{91}$. Però, ci sono differenze effettive tra i due autori.

Rispetto a queste differenze, in sintesi, Kant fonda la moralità sull'autonomia della volontà (ragione pratica) la quale cerca di colmare due caratteristiche fondamentali, la necessità e l'universalità in base ai principi a priori della stessa ragione. Anche la facoltà di desiderare è sottomessa ai principi della ragione ${ }^{92}$. Levinas, invece, fonda la moralità sull'eteronomia che viene dall' "elezione" nel Bene. È a partire da questa fonte "diacronica" che Levinas pensa la fraternità e non l'universalità astratta.

Kant alla fine della Fondazione della metafisica dei costumi conclude dicendo che crede in due cose: nella morale dentro di sé e nel cielo stellato sopra di sé. Levinas arriva alla fine di Altrimenti che essere affermando 1'“esteriorità per eccellenza" nel senso di altezza e cielo: "Il regno del cielo è etico" 93 .

\footnotetext{
${ }^{86}$ Cf. CHALIER, 1998, p. 82.

${ }^{87}$ Questo modo di dire viene dalla spirazione "profetica". Levinas cita, per esempio, il testo del profeta Isaia 6,8: eccomi! (Cf. LEVINAS, 2011, p. 183).

88 "Contrairement à Kant, Levinas ne pense pas que le sujet soit moral et libre en raison de son autonomie, mais en raison de cette élection” (CHALIER, 1998, p. 94).

${ }^{89}$ LEVINAS, 2011, p. 182.

${ }^{90}$ Ibidem, p. 154-155.

${ }^{91}$ Cf. CHALIER, 2011, p.10-11.

${ }^{92}$ Cf. Ibidem, p. 110.

${ }^{93}$ LEVINAS, 2011, p. 227. Per un lavoro più critico sarebbe molto interessante continuare il confronto con Kant a partire dall'opera La religione nei limiti della semplice ragione (1793), dove lui sviluppa il concetto di "regno di Dio".
} 


\section{Riferimenti}

CHALIER, C. Judaisme et alterite. Lagrasse: Verdier, 1982.

. Pour une morale au-delà du savoir. Kant et Levinas. Paris: Albin Michel, 1998.

D'ANTUONO, E.; KAJON, I.; SINDONI, P.R. Giacobbe e l'angelo: figure ebraiche alle radici della modernità europea. Roma: Lithos, 2012.

DERRIDA, J. Addio a Emmanuel Levinas. Milano: Jaca Book, 2011.

GATES, D.C. "The Fact of Reason and the Face of the Other: Autonomy, Constraint, and Rational Agency in Kant and Levinas", The Southern Journal of Philosophy, v.40, n.4, 2002, 493-522.

HUSSERL, E. Meditazioni cartesiane. Bergamo: Bompiani, 2002.

KANT, I. Critica della ragion pura. Milano: Bompiani, 2007.

. Fondazione della metafisica dei costumi. Bari: Laterza, 2009.

LA SACRA BIBBIA. Città del Vaticano: CEI - Libreria Editrice Vaticana, 2008.

LEVINAS, E. Il tempo e l'altro. Genova: Il Melangolo, 1993.

Scoprire l'esistenza con Husserl e Heidegger. Milano: Raffaello Cortina, 1998.

. Umanesimo dell'altro uomo. Genova: Il Melangolo, 2009.

. Altrimenti che essere o al di là dell'essenza. Como: Jaca Book, 2011.

Totalità e infinito. Saggio sull'esteriorità. Foligno: Jaca Book, 2012.

. Etica e infinito: dialoghi con Philippo Nemo. Roma: Castelvecchi, 2012.

LEVINAS, E.; CASPER, B. In ostaggio per l'altro. Pisa: ETS, 2012.

NIETZSCHE, F. Genealogia della morale. Milano: Adelphi, 1984.

PASCAL, G. O pensamento de Kant. Petrópolis: Vozes, 2001.

RENAUT, A. "Levinas et Kant". In: J.-L. MARION, J.-L. (Org.). Emmanuel Levinas, Positivité et transcendence. Paris: Presses universitaires de France, 2000, 89-104.

SESTA, L. La legge dell'altro. La fondazione dell'etica in Levinas e Kant. Pisa: ETS, 2005. 